Supporting Information

\title{
Biomimetic Mechanically Strong One-Dimensional Hydroxyapatite/Poly(D,L lactide) Composite Inducing Formation of Anisotropic Collagen Matrix
}

Yonggang Zhang ${ }^{\dagger}$, Jiaping $\mathrm{Li}^{\dagger, \dagger}$, Vivian Hilda Maria Mouser", Nadia Roumans ${ }^{\S}$, Lorenzo Moroni $^{\ddagger}$, Pamela Habibovic* $* \dagger$

$\dagger$ Department of Instructive Biomaterials Engineering, Maastricht University, MERLN Institute for Technology-Inspired Regenerative Medicine, Universiteitssingel 40, 6229 ER, Maastricht, The Netherlands

† Department of Complex Tissue Regeneration, Maastricht University, MERLN Institute for Technology-Inspired Regenerative Medicine, Universiteitssingel 40, 6229 ER, Maastricht, The Netherlands

$\S$ Department of Cell Biology-Inspired Tissue Engineering, Maastricht University, MERLN Institute for Technology-Inspired Regenerative Medicine, Universiteitssingel 40, 6229 ER, Maastricht, The Netherlands

$\|$ Orthopaedic Biomechanics, Department of Biomedical Engineering, Eindhoven University of Technology, PO Box 513, 5600 MB Eindhoven, The Netherlands

E-mail: p.habibovic@maastrichtuniversity.nl

\section{Extended Methods}

Materials. Anhydrous calcium chloride (96\%, Thermos Fisher), strontium chloride hexahydrate (>99\%, Boom), copper chloride (Sigma-Aldrich), sodium hydroxide (98\%, Sigma 
Aldrich), sodium hexametaphosphate (extra pure, Thermos Fisher), oleic acid (pure, Thermos Fisher), polycaprolactone (PCL, Mn 80000, Sigma-Aldrich), poly(DL-lactide) (PDLA, PURASORB $®$ PDL 05, Corbion), chloroform ( $\geqslant 98 \%$, VWR), acetone ( $\geqslant 99 \%$, VWR), HA nanopowder (Merck), tris(hydroxymethyl)aminomethane (Tris, VWR), and ethylenediaminetetraacetic acid disodium salt dihydrate (EDTA, Sigma Aldrich).

Synthesis of Stoichiometric 1D HA (1D HA), 1D Sr-HA and 1D Cu-HA. For synthesis of stoichiometric 1D HA, $56 \mathrm{~mL}$ of ethanol, $64 \mathrm{~mL}$ of oleic acid and $40 \mathrm{~mL}$ of milliQ water were mixed for 15 min using a magnetic stirrer. $80 \mathrm{~mL}$ of $\mathrm{CaCl}_{2}$ aqueous solution $(0.2 \mathrm{M})$ was added into this ternary solvent system, followed by the addition of $80 \mathrm{~mL} \mathrm{NaOH}$ aqueous solution $(1.65 \mathrm{M}) 15 \mathrm{~min}$ later. Then, $1 \mathrm{~h}$ after the addition of $\mathrm{NaOH}$ aqueous solution, $80 \mathrm{~mL}$ of $\left(\mathrm{NaPO}_{3}\right)_{6}$ aqueous solution $(0.044 \mathrm{M})$ was added and stirred for $30 \mathrm{~min}$. The above reaction system was transferred into a $500 \mathrm{~mL}$ Teflon-lined stainless steel autoclave, placed in an oven and maintained at $180{ }^{\circ} \mathrm{C}$ for $36 \mathrm{~h}$. Finally, the autoclave was cooled to room temperature, the product was collected, washed with ethanol and milliQ water, and dried. 1D HA was treated in a muffle furnace (Nabertherm L16/14, Germany) at $500{ }^{\circ} \mathrm{C}$ for $3 \mathrm{~h}$ at a heating rate of $5{ }^{\circ} \mathrm{C}$ $\min ^{-1}$. 1D Sr-HA was synthesized using the same method as for 1D HA, with the exception that instead of $80 \mathrm{~mL}$ of $\mathrm{CaCl}_{2}$ aqueous solution $(0.2 \mathrm{M}), 80 \mathrm{~mL}$ of mixed aqueous solution consisting of $\mathrm{CaCl}_{2}(0.183 \mathrm{M})$ and $\mathrm{SrCl}_{2} \cdot 6 \mathrm{H}_{2} \mathrm{O}(0.025 \mathrm{M})$ was used. 1D Cu-HA synthesis also followed the same method, except that instead of $80 \mathrm{~mL}$ of $\mathrm{CaCl}_{2}$ aqueous solution, $80 \mathrm{~mL}$ of mixed aqueous solution consisting of $\mathrm{CaCl}_{2}(0.193 \mathrm{M})$ and $\mathrm{CuCl}_{2}(0.01 \mathrm{M})$ was used with a $\mathrm{pH}$ of 4.4 adjusted with $1 \mathrm{M} \mathrm{HCl}$. The doping amount of $\mathrm{Sr}$ and $\mathrm{Cu}$ ions was based on previous work. $^{1,2}$ 
The specific surface area of the Nano HA and undoped 1D HA was determined using the Brunauer-Emmett-Teller (BET) method on a specific surface area analyzer (Micromeritics ASAP 2060, Germany).

Swelling Properties of Sr/Cu-doped 1D HA/PDLA Porous Scaffolds. Conventional simulated body fluid $(1 \times \mathrm{SBF})$ was prepared according to a previous report. ${ }^{3}$ Above fabricated $\mathrm{Sr} / \mathrm{Cu}$-doped 1D HA/PDLA porous scaffold $\left(5.7 \times 5.7 \times 3 \mathrm{~mm}^{3}, \mathrm{n}=3\right.$ for each time point $)$ with a porosity of $50 \%$, was placed into $10 \mathrm{~mL}$ of SBF solution inside a $50 \mathrm{~mL}$ centrifugation tube

at $37{ }^{\circ} \mathrm{C}$. At day 4, 7 and 14 , the dimensions of the porous scaffolds were measured. The volumetric swelling ratio was calculated by the following equation:

$$
\text { Swelling ratio }(\%)=\left(\mathrm{V}_{\mathrm{x}}-\mathrm{V}_{\text {original }}\right) \times 100 \% / \mathrm{V}_{\text {original }}
$$

where $V_{x}$ is the volume of the porous scaffold at day $x\left(x=4,7\right.$ and 14) and $V_{\text {original }}$ is the initial volume of the porous scaffold. Porous PDLA scaffold and Nano HA/PDLA scaffolds with the same dimension and porosity were used as controls.

\section{In Vitro Mechanical Stability of Sr/Cu-doped 1D HA/PDLA Porous Scaffolds. hMSCs} from one donor, obtained after written informed consent, were expanded in basic cell culture medium containing $\alpha$-MEM (Gibco) with $0.2 \mathrm{mM}$ ascorbic acid (Sigma), $100 \mathrm{U} \mathrm{mL}^{-1}$ penicillin, $100 \mathrm{mg} \mathrm{mL}^{-1}$ streptomycin (Gibco) and 10\% FBS (Sigma). Upon reaching confluency, the cells were frozen for further use. For experiment described here, hMSCs were used at passage 5 and 6. To investigate the in vitro mechanical stability, $100 \mu \mathrm{L}$ of concentrated hMSCs suspension $\left(100 \times 10^{4} / \mathrm{mL}\right)$ in basic cell culture medium was placed on top of the $\mathrm{Sr} / \mathrm{Cu}$-doped $1 \mathrm{D}$ HA/PDLA porous scaffolds $\left(5.7 \times 5.7 \times 3 \mathrm{~mm}^{3}\right)$ with porosity of $50 \%$ inside 24 -well plates. The suspension infiltrated the scaffold pores within several seconds. After incubation in an incubator for $4 \mathrm{~h}$, another $1.4 \mathrm{~mL}$ basic cell culture medium was added to each well. Medium 
refreshment was done every 3 days. At day 14, the scaffolds were harvested and dried, and the compressive strength of the scaffolds was tested by using a material testing machine (Zwick/Roell, Germany) as described in the Experimental Section in the main article. Porous PDLA scaffolds and Nano HA/PDLA scaffolds with the same dimension and porosity were used as controls.

In Vitro Mineralization. Sr/Cu-doped 1D HA (25 wt \%)/PDLA (75 wt \%) scaffold $(5.7 \times 5.7$ $\times 6 \mathrm{~mm}^{3}, \mathrm{n}=3$ for each time point) with porosity of $50 \%$ was placed into $10 \mathrm{~mL}$ of SBF solution inside a $50 \mathrm{~mL}$ centrifugation tube. The tube was sealed and placed in a water bath at the temperature of $37^{\circ} \mathrm{C}$. SBF solution was refreshed every two days. At day 7, the samples were collected from the tube, washed with distilled water and dried. The scaffolds were sputtercoated with gold and analyzed using SEM (Philips XL30, the Netherlands). Porous PDLA and Nano HA/PDLA porous scaffolds were used as controls.

In Vitro Ion Release. Sr/Cu-doped 1D HA/PDLA porous scaffold $\left(5.7 \times 5.7 \times 6 \mathrm{~mm}^{3}, \mathrm{n}=5\right.$ for each time point) with porosity of $50 \%$, was placed into $10 \mathrm{~mL}$ of physiological saline solution $(\mathrm{NaCl}, 0.9 \% \mathrm{w} / \mathrm{v})$ inside a $50 \mathrm{~mL}$ centrifugation tube. The tube was sealed and placed in a water bath at the temperature of $37^{\circ} \mathrm{C}$. At day of $1,4,7,10,16,21,26,31$, and 36 , a $0.5 \mathrm{~mL}$-sample was collected from the tube, and replaced by $0.5 \mathrm{~mL}$ of fresh saline solution. Finally, the ion content of the collected samples was analyzed using inductively coupled plasma mass spectrometry (ICP-MS, Thermo Fisher SCIENTIFIC iCAP Q, Germany).

Cell Culture. The cell seeding method used for investigating attachment, viability, proliferation and ECM formation, was the same as described for the in vitro mechanical stability test. To study the ALP activity of hMSCs, the same seeding method was used with the exception 
that the osteogenic cell culture medium (basic medium with $10 \mathrm{nM}$ dexamethasone) was used instead of the basic cell culture medium. Porous PDLA and Nano HA/PDLA scaffolds with the same dimension and porosity were used as controls.

Live/Dead Staining. For live/dead assay, after 3 days of culture, porous scaffolds (n=2) with cells were washed with phosphate-buffered saline (PBS) solution, and stained with CalceinAM (Fisher Scientific) and ethidium homodimer-1 (EthD-1) (Fisher Scientific) in PBS for 30 min. The samples were washed with PBS and then observed using a fluorescence microscope (Nikon Eclipse Ti-S, Japan).

Cell Metabolic Activity. The metabolic activity of cells on scaffolds ( $n=4)$ was measured by using PrestoBlue kit (Thermo Fisher Scientific). At day 7 and day 14, culture medium was removed from wells, and the cells were washed with $700 \mu \mathrm{L}$ of PBS. Then, PBS was removed and $700 \mu \mathrm{L}$ of PrestoBlue ${ }^{\mathrm{TM}}$ solution (10\% in culture medium) was added to each well. After incubation for $30 \mathrm{~min}$ at $37{ }^{\circ} \mathrm{C}$ in the dark, $100 \mu \mathrm{L}$ of incubated solution was transferred to a new black 96-well plate. The fluorescence was measured on the microplate reader (BMG Labtech CLARIOstar, Germany) at $590 \mathrm{~nm}$.

Quantification of DNA. CyQUANT ${ }^{\mathrm{TM}}$ Cell Proliferation Assay (Fisher Scientific) was used to determine the proliferation of hMSCs on scaffolds ( $n=4)$. At day 7 and day 14, scaffolds with cells were washed with PBS and freeze-thawed at $-80{ }^{\circ} \mathrm{C}$ and room temperature, respectively 3 times. Subsequently, the cells were incubated with proteinase K (Sigma) solution in Tris/EDTA buffer overnight at $56{ }^{\circ} \mathrm{C}$. Then RNase buffer was added to degrade the cellular RNA. Finally, $100 \mu \mathrm{L}$ of solution was transferred to a new black 96-well plate, followed by the addition of $100 \mu \mathrm{L}$ of GR-dye. After incubation for $15 \mathrm{~min}$ in the dark at room temperature, the 
fluorescence intensity of the solution was measured using the microplate reader (BMG Labtech CLARIOstar, Germany) at $480 \mathrm{~nm}$ (excitation) and $520 \mathrm{~nm}$ (emission).

Cell Morphology. After 3 and 7 days of culture, scaffolds $(n=2)$ with cells were washed with PBS and fixed overnight by using $4 \% \mathrm{w} / \mathrm{v}$ formaldehyde (Sigma) at $4{ }^{\circ} \mathrm{C}$. After treatment with $0.1 \% \mathrm{v} / \mathrm{v}$ Triton X-100 (VWR) and $1 \mathrm{wt} \%$ bovine serum albumin (BSA, VWR), the cells were stained using Alexa Fluor 488 phalloidin (Fisher Scientific) and DAPI (Sigma-Aldrich). Finally, the morphology of cells on scaffold was observed using a fluorescence microscopy (Nikon Eclipse Ti-S, Japan). For SEM observation, the cells were dehydrated by using a series of ethanol concentrations $(30 \%, 50 \%, 70 \%, 90 \%, 95 \%$ and 100\%). Finally, the cells were dried in fume hood and sputter-coated with gold and observed using a SEM (Philips XL30, the Netherlands).

Alkaline Phosphatase (ALP) Activity. After 7 and 14 days of culture in osteogenic culture medium (basic culture medium with $10 \mathrm{nM}$ dexamethasone), the ALP activity of cells cultured on scaffolds ( $n=4)$ was quantified by using the CDP-Star kit (Roche). Briefly, scaffolds with cells were washed with PBS three times, and incubated with lysis buffer $\left(0.1 \mathrm{M} \mathrm{KH} \mathrm{KHO}_{4}, 0.1 \mathrm{M}\right.$ $\mathrm{K}_{2} \mathrm{HPO}_{4}$ and $0.1 \%$ Triton X-100) for $1 \mathrm{~h}$ while shaking. $40 \mu \mathrm{L}$ of solution was transferred to a white 96-well plate, followed by the addition of $160 \mu \mathrm{L}$ of CDP-Star reagent. After incubation for $15 \mathrm{~min}$ at room temperature, the luminescence was measured at $466 \mathrm{~nm}$ by the microplate reader (BMG Labtech CLARIOstar, Germany). The ALP values were normalized to DNA content from the same sample.

Collagen Immunostaining. After 14 days of culture, scaffolds $(n=4)$ with cells were washed with PBS and fixed with $4 \% \mathrm{w} / \mathrm{v}$ formaldehyde for $20 \mathrm{~min}$. After fixation, the cells were 
incubated with $0.1 \%$ Triton X-100 for 10 min, followed by blocking with blocking solution (1\% BSA in PBS) for $1 \mathrm{~h}$ at room temperature. Then the cells were incubated with primary antibody (Anti-Collagen I antibody, ab34710, Abcam) overnight at $4{ }^{\circ} \mathrm{C}$. After treatment with secondary antibody (Goat anti-Rabbit Alexa Fluor 488, Fisher Scientific) for 1h, the cells were stained using Alexa Fluor 647 phalloidin (Fisher Scientific) and DAPI (Sigma-Aldrich). Fluorescence images were captured by a fluorescence microscope (Nikon Eclipse Ti-S, Japan).

Statistical Analysis. For each test, two independent experiments were performed, and the results of one representative experiment are shown in the manuscript. Statistical analysis was performed using GraphPad Prism 9 (GraphPad software, San Diego, USA). Values are expressed as means \pm standard deviation (STDEV). Statistical differences were determined by the analysis of One-Way ANOVA with Tukey's multiple comparison tests. The differences were considered statistically significant at $\mathrm{p}<0.05$, and the data were indicated with $(*)$ for $\mathrm{p}<0.05,(* *)$ for $\mathrm{p}<0.01,(* * *)$ for $\mathrm{p}<0.001$, and $(* * * *)$ for $\mathrm{p}<0.0001$, respectively. 


\section{Supplementary Figures}
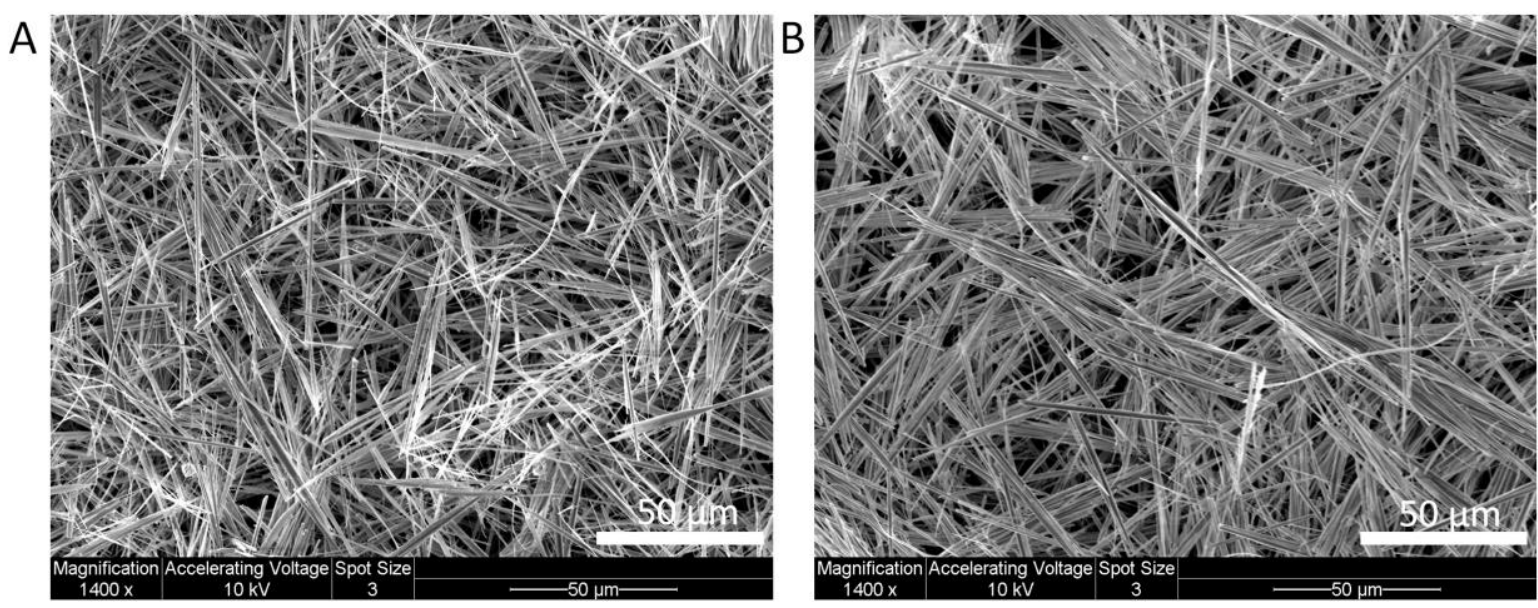

C

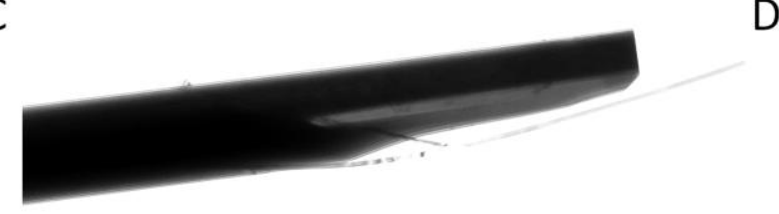

D
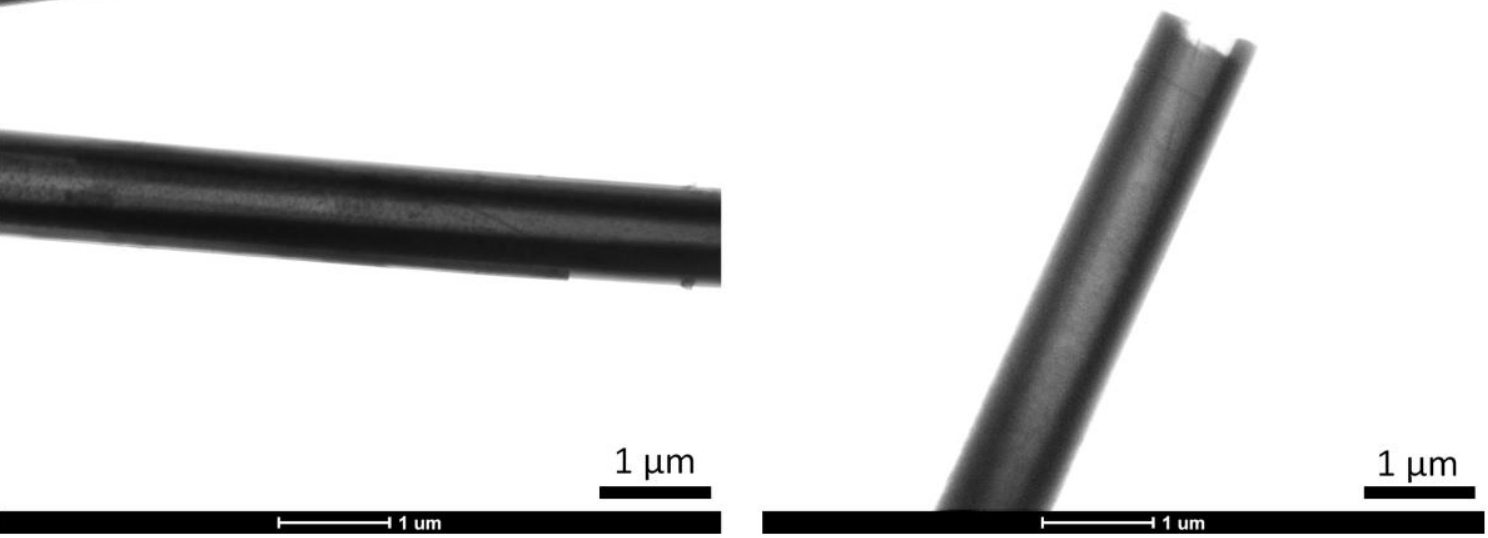

E
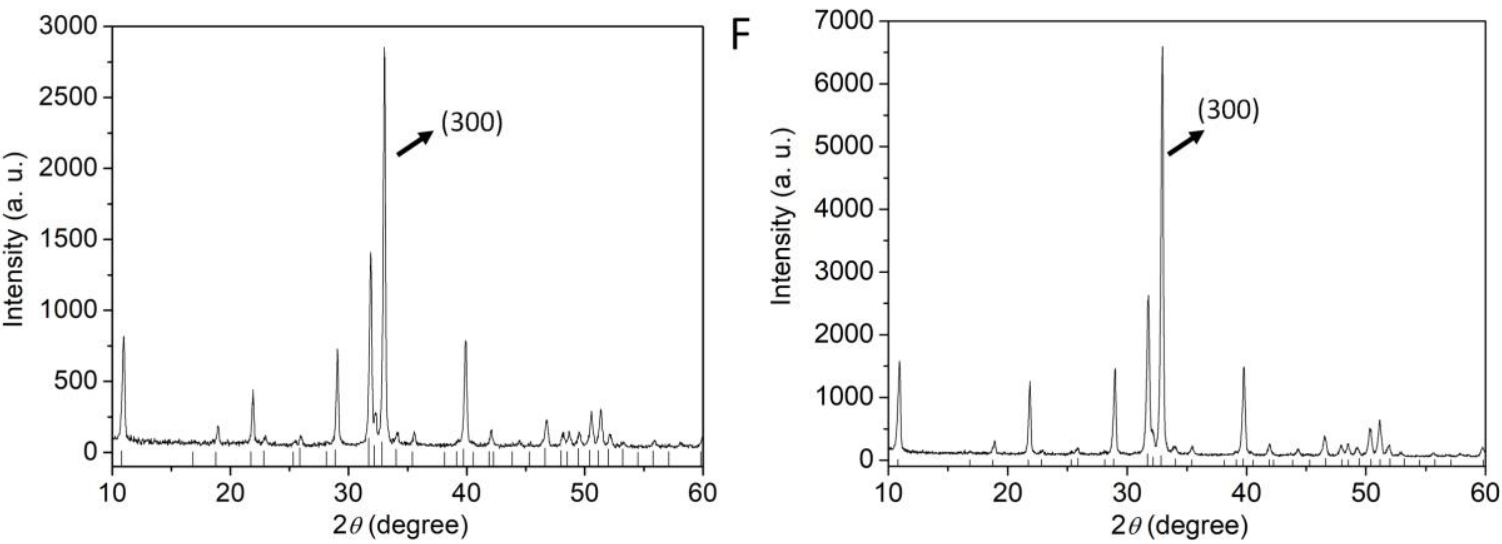

Figure S1. (A,B) SEM micrographs, (C,D) TEM micrographs and (E,F) XRD patterns of 1D $\mathrm{Cu}-\mathrm{HA}$ and 1D Sr-HA, respectively. 

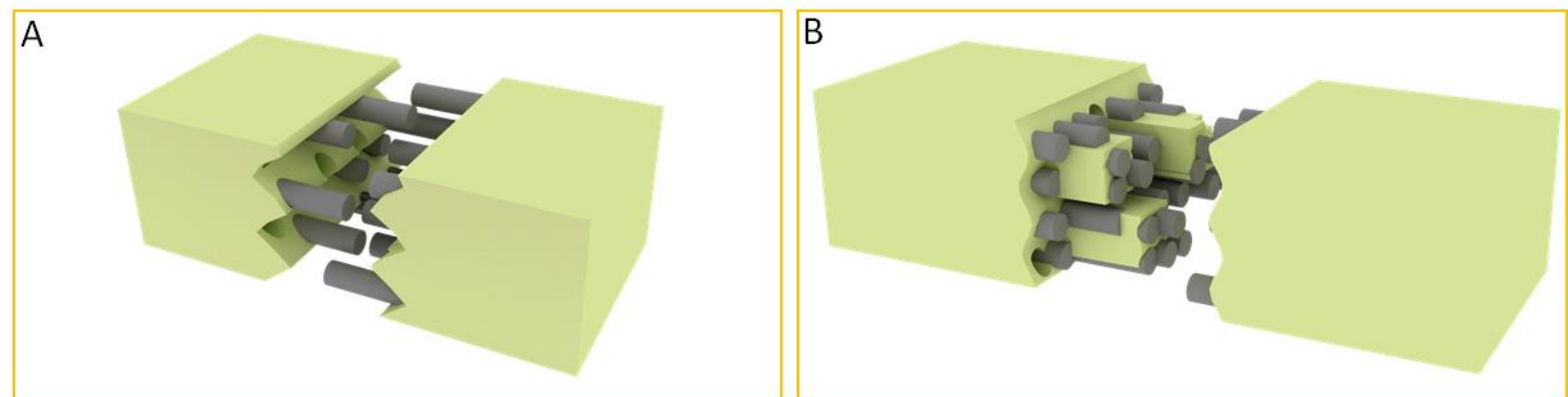

Figure S2. Schematic illustration of the fracture model of the biomimetic Sr/Cu-doped 1D

HA/PCL composite with 30 wt \% Sr/Cu-doped 1D HA (A) and 50 wt \% Sr/Cu-doped 1D HA (B), respectively. 
A
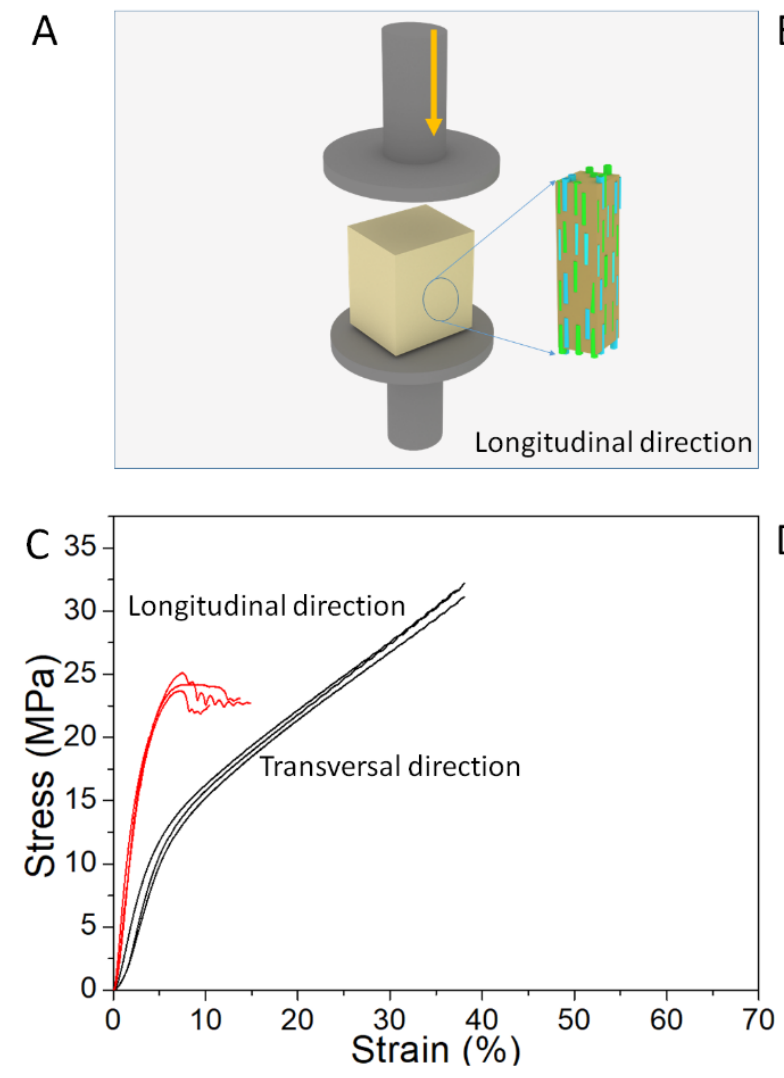

B
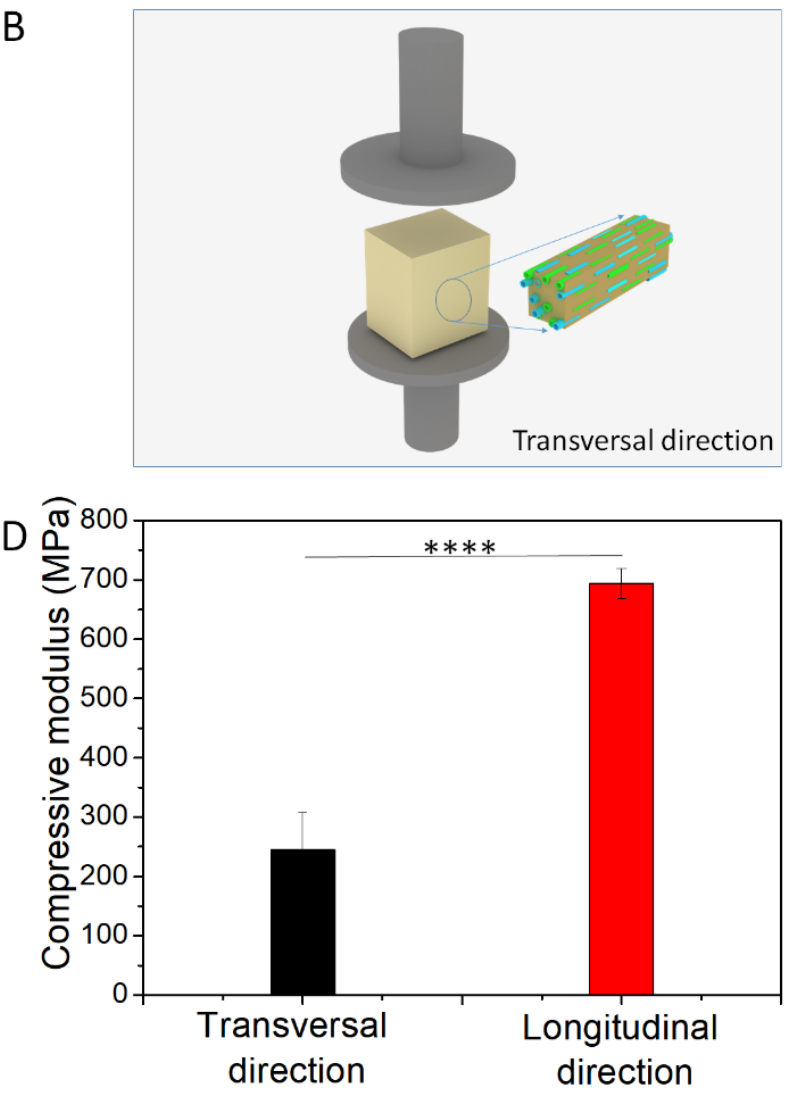

Figure S3. (A and B) Schematic illustration of the loading process in the two different directions. (C) Compressive stress-strain curves of the biomimetic $\mathrm{Sr} / \mathrm{Cu}$-doped 1D HA/PCL composite with $30 \mathrm{wt} \% \mathrm{Sr} / \mathrm{Cu}$-doped 1D HA in longitudinal and transversal direction. (D) Compressive modulus of the biomimetic Sr/Cu-doped 1D HA/PCL composite with 30 wt \% $\mathrm{Sr} / \mathrm{Cu}$-doped 1D HA in longitudinal and transversal direction. $\left(^{*}\right)$ for $\mathrm{p}<0.05$, $\left({ }^{* *}\right)$ for $\mathrm{p}<0.01$, $(* * *)$ for $\mathrm{p}<0.001$, and $(* * * *)$ for $\mathrm{p}<0.0001$, respectively. 

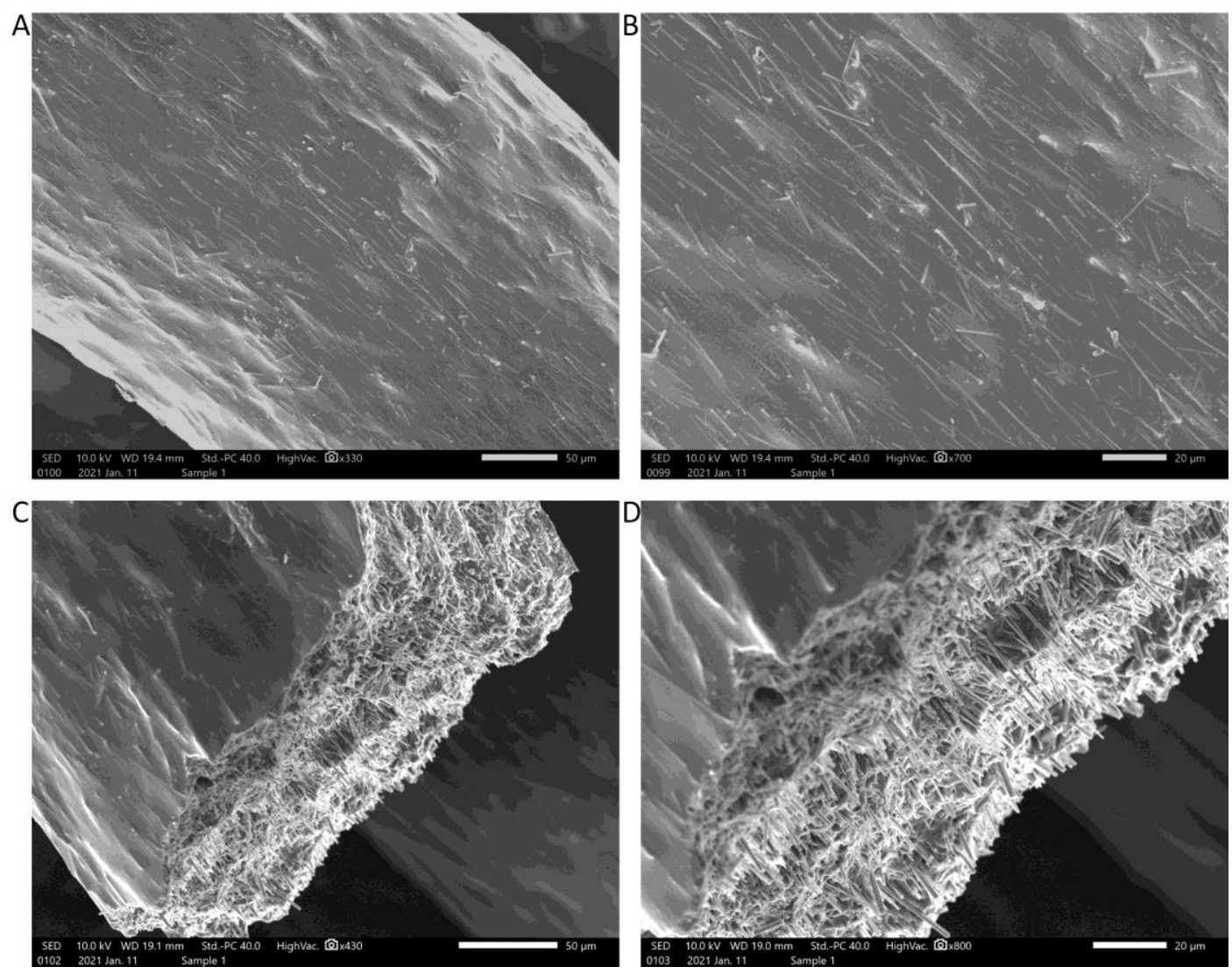

Figure S4. SEM micrographs of the biomimetic Sr/Cu-doped 1D HA/PDLA with 25 wt \% $\mathrm{Sr} / \mathrm{Cu}$-doped 1D HA. 


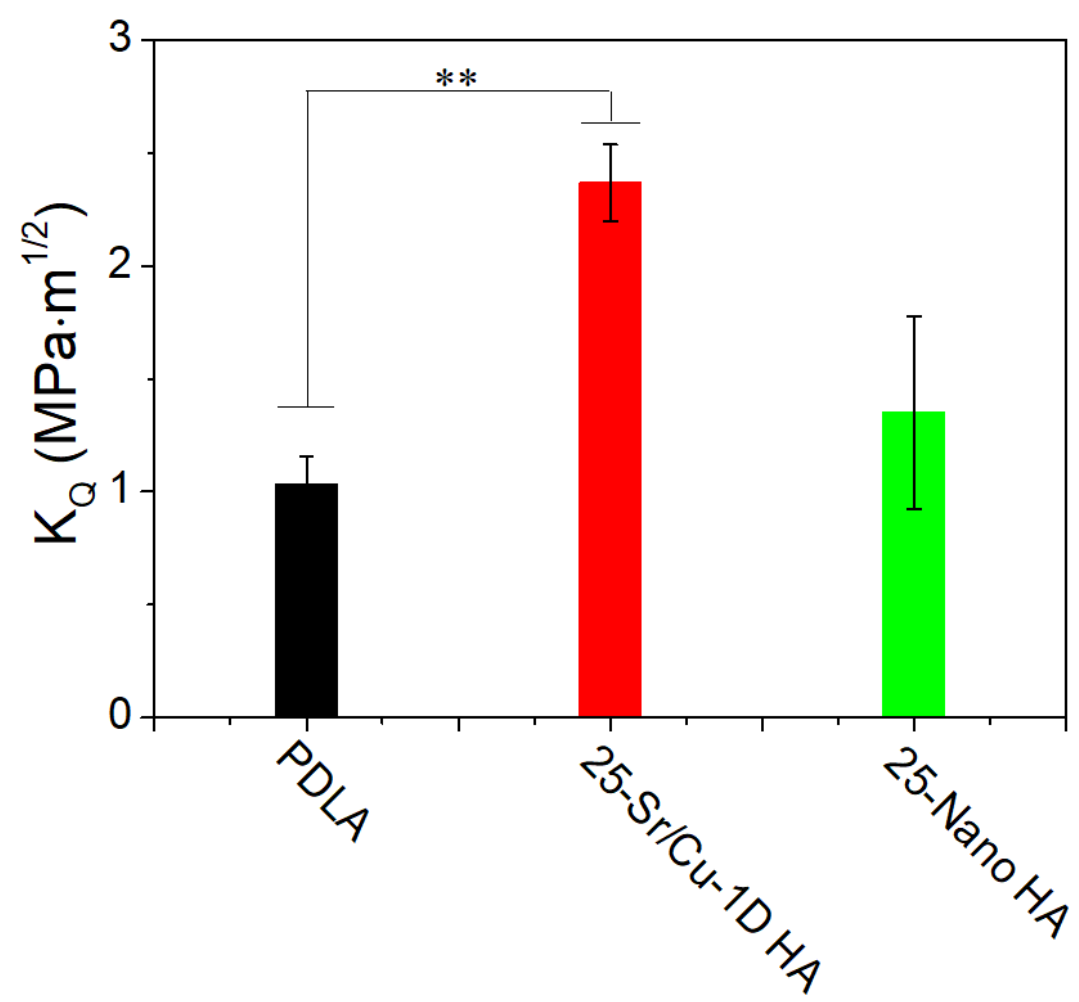

Figure S5. Fracture toughness $\left(\mathrm{K}_{\mathrm{Q}}\right)$ of pure PDLA, biomimetic Sr/Cu-doped 1D HA/PDLA composite with 25 wt \% Sr/Cu-doped 1D HA, and Nano HA/PDLA composite with 25 wt \% Nano HA, respectively. (*) for $\mathrm{p}<0.05,(* *)$ for $\mathrm{p}<0.01,(* * *)$ for $\mathrm{p}<0.001$, and $(* * * *)$ for $\mathrm{p}<0.0001$, respectively. 


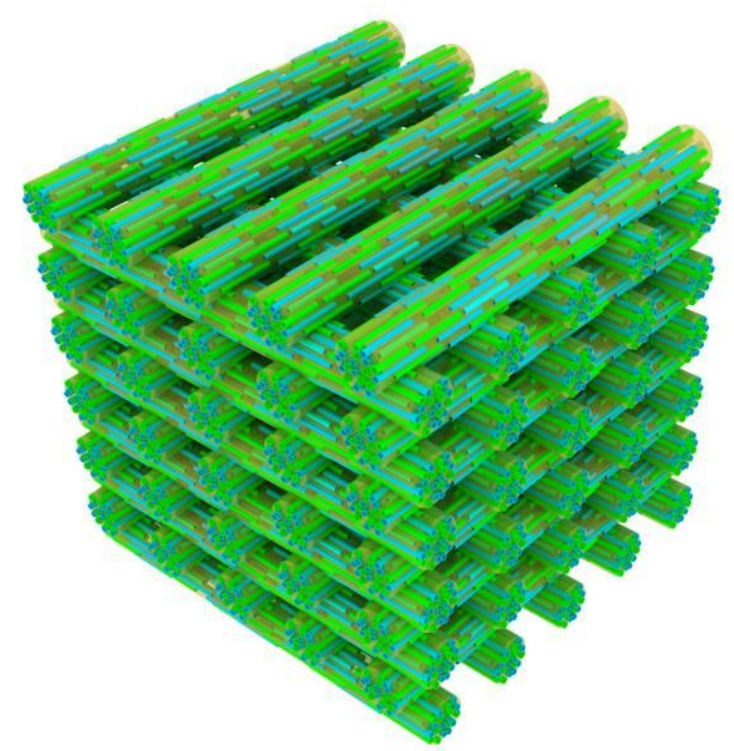

Figure S6. Schematic illustration of the structure of Sr/Cu-doped 1D HA/PDLA porous scaffolds. 

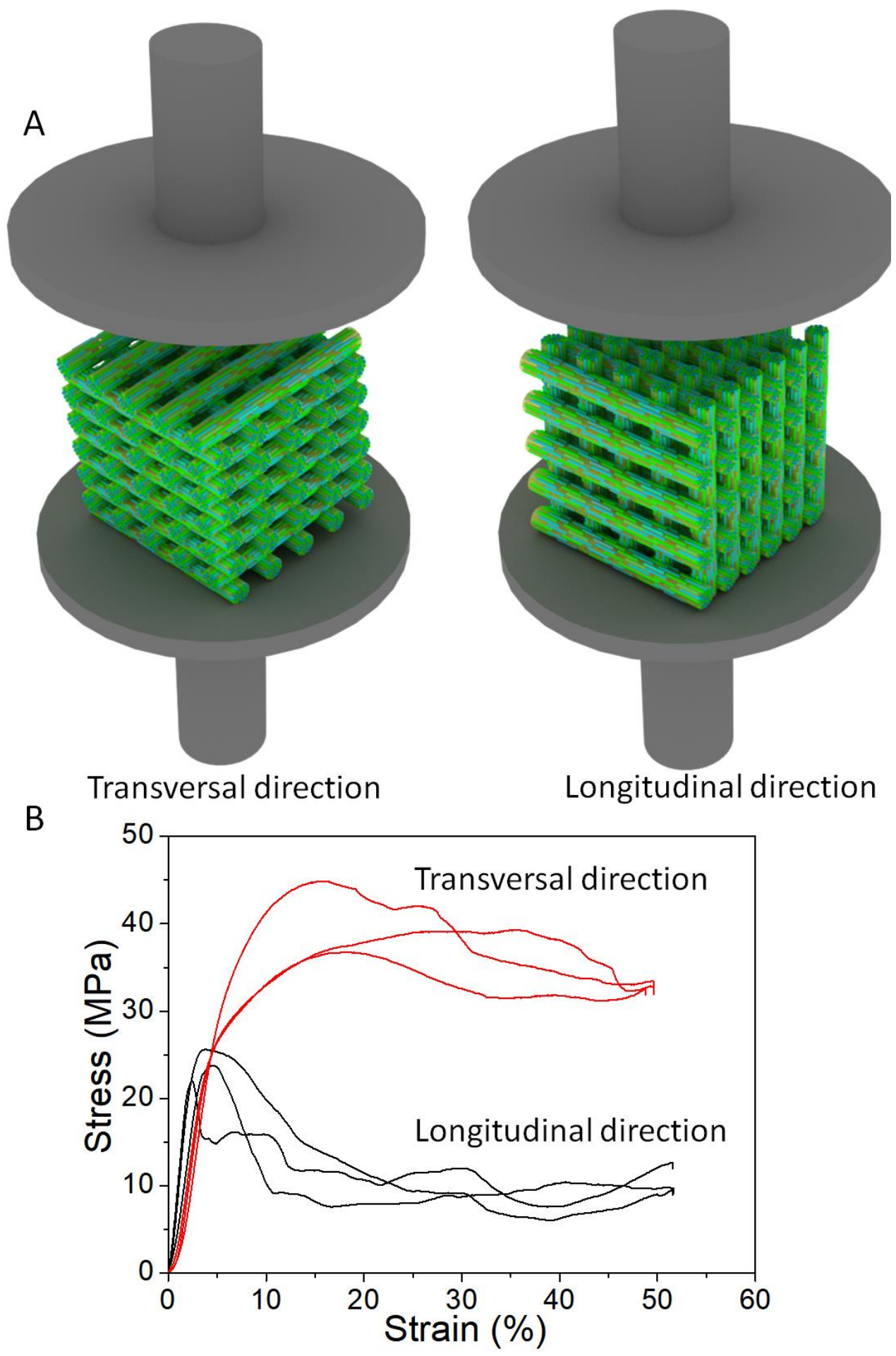

Figure S7. (A) Schematic illustration of the compression tests of the $\mathrm{Sr} / \mathrm{Cu}$-doped 1D HA/PDLA porous scaffolds in two different directions. (B) Compressive stress-strain curves of the $\mathrm{Sr} / \mathrm{Cu}$-doped 1D HA/PDLA porous scaffolds in longitudinal and transversal direction. 


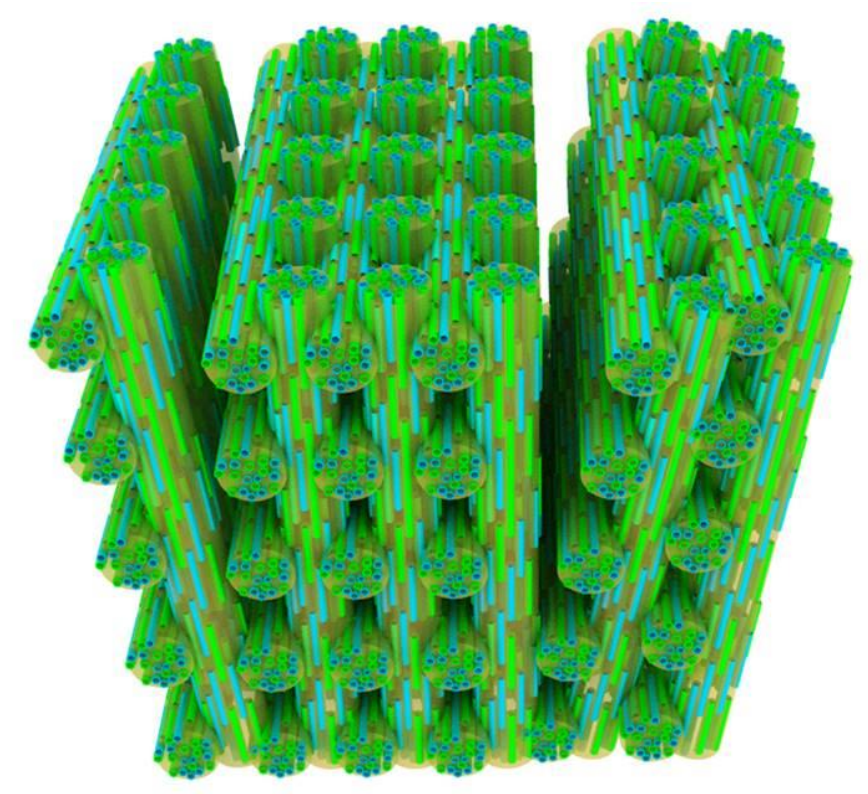

Figure S8. Schematic illustration of the detachment of the layers of the $\mathrm{Sr} / \mathrm{Cu}$-doped 1D HA/PDLA porous scaffolds during compression test in longitudinal direction. 


\section{Pure PDLA porous scaffold}

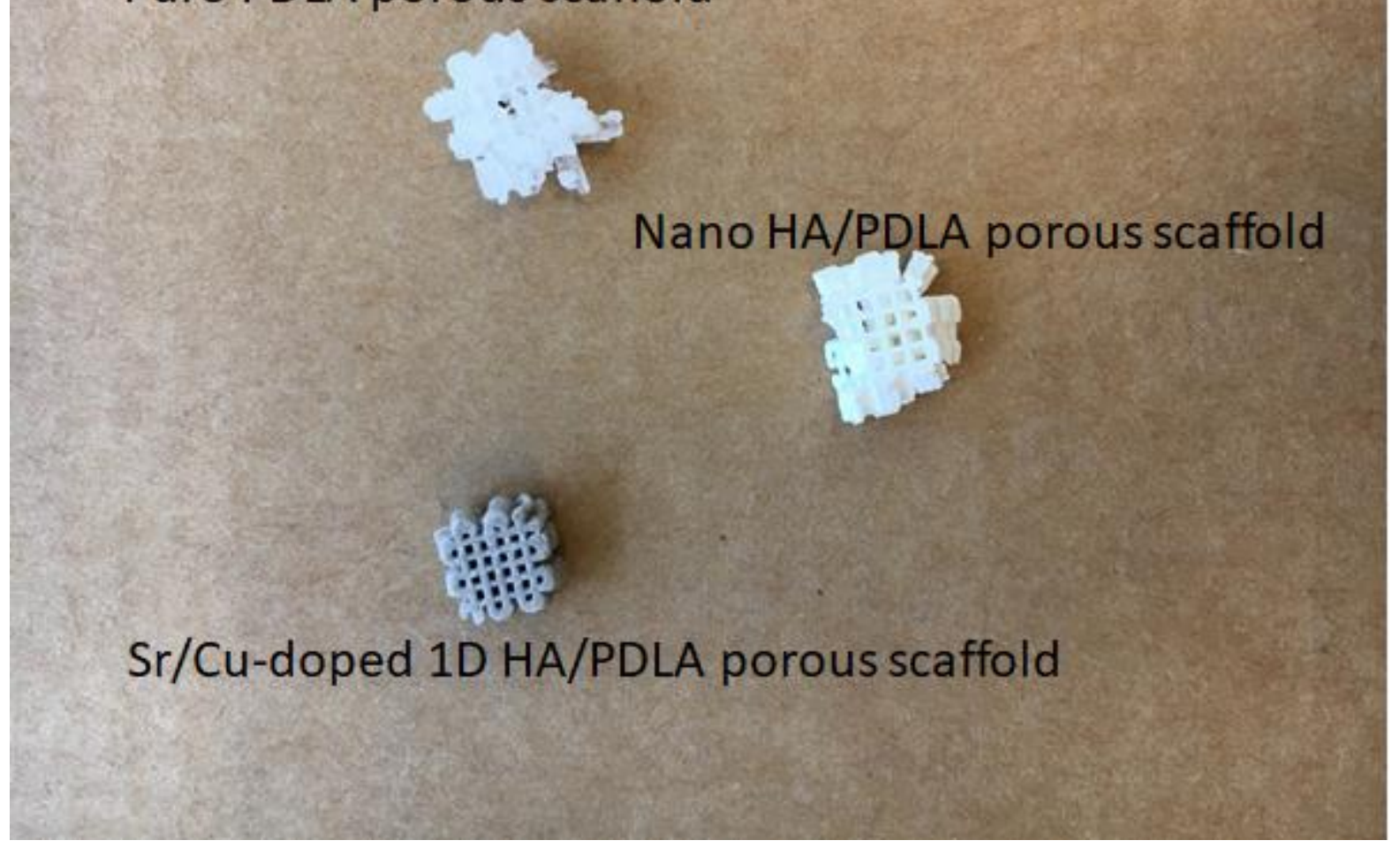

Figure S9. Digital images of the Sr/Cu-doped 1D HA/PDLA porous scaffolds, pure PDLA porous scaffolds and Nano HA/PDLA porous scaffolds after compression test in transversal direction, respectively. 

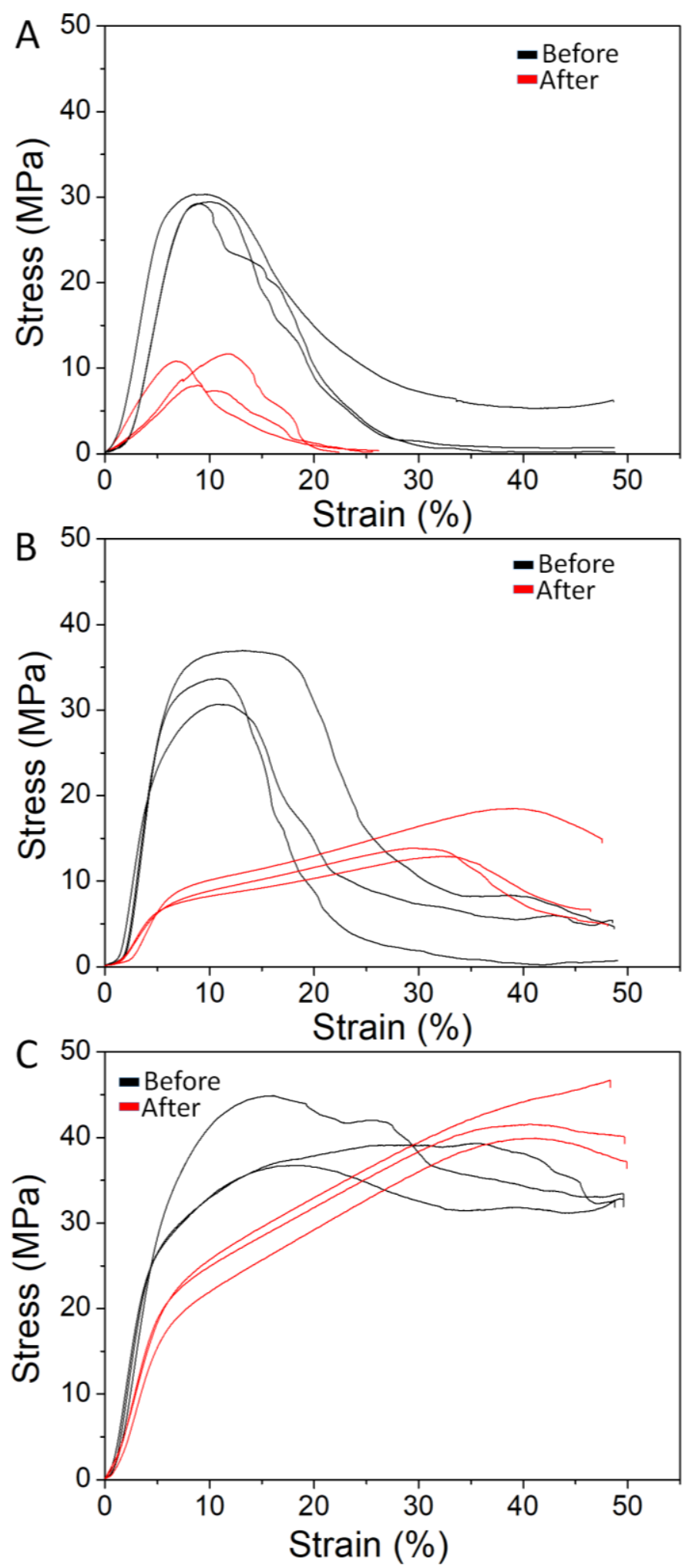

Figure S10. (A to C) Compressive stress-strain curves of the pure PDLA porous scaffolds (A), Nano HA/PDLA porous scaffolds (B) and Sr/Cu-doped 1D HA/PDLA porous scaffolds (C) before and after 14-day hMSCs culture. 


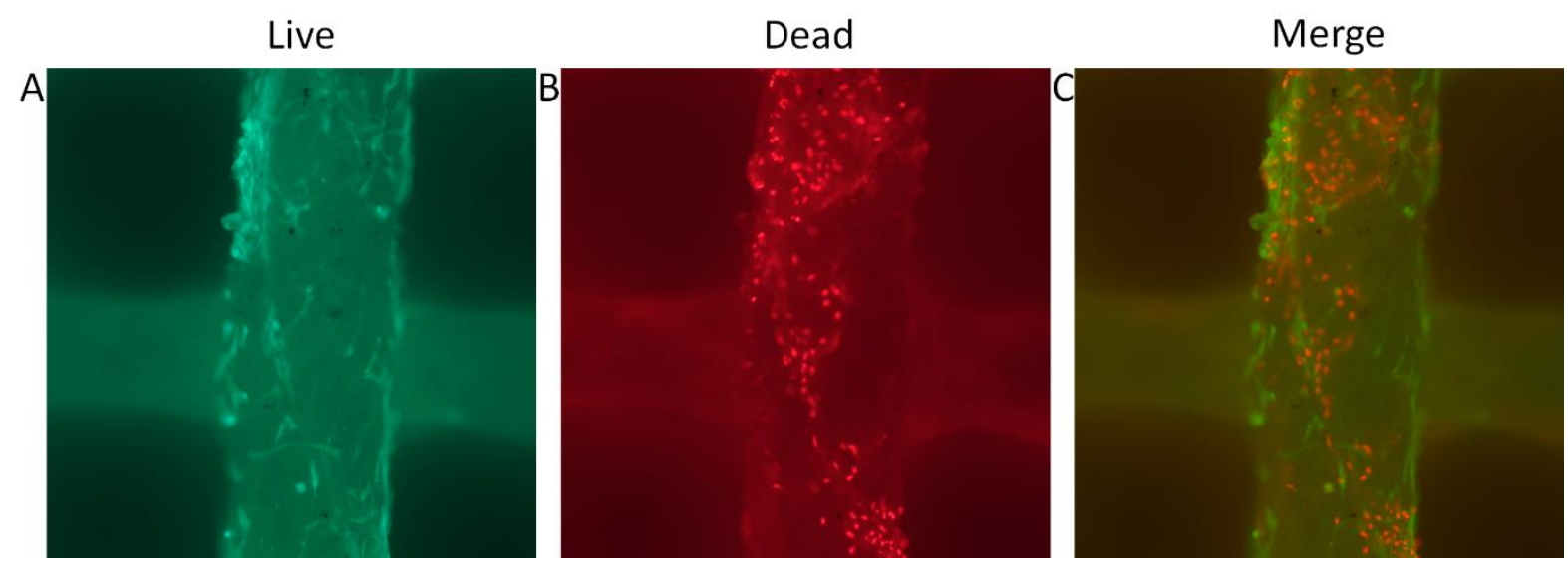

Figure S11. (A to C) Fluorescence microscopy images of live (stained green) and dead (stained red) hMSCs cultured for 3 days on the $\mathrm{Sr} / \mathrm{Cu}$-doped 1D HA/PDLA porous scaffolds. 

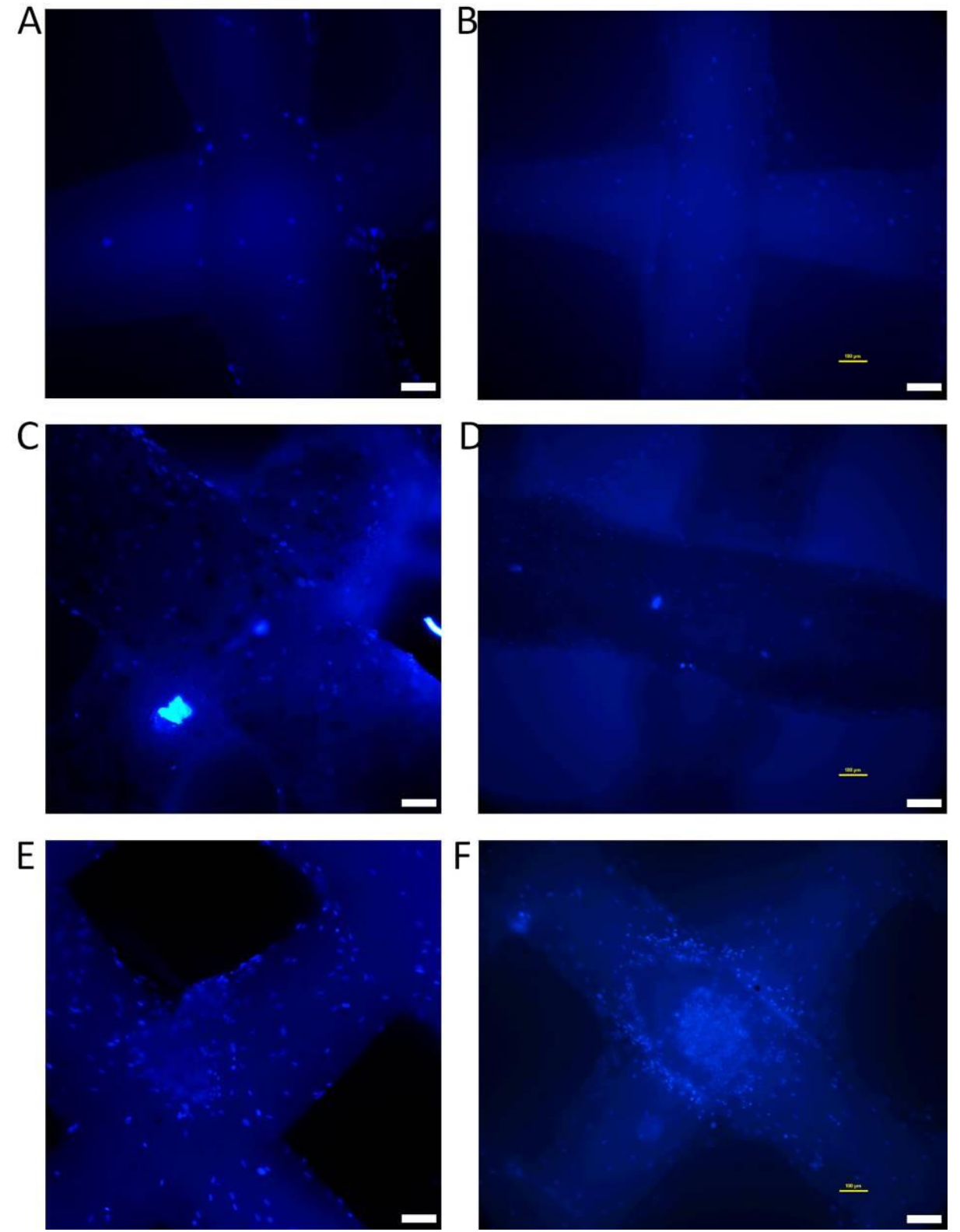

Figure S12. Fluorescence microscopy images of nuclei (DAPI: blue) of hMSCs cultured on (A,B) pure PDLA porous scaffolds, (C,D) Nano HA/PDLA porous scaffolds and (E,F) Sr/Cudoped 1D HA/PDLA porous scaffolds for 3 and 7 days, respectively. Scale bar: $100 \mu \mathrm{m}$. 


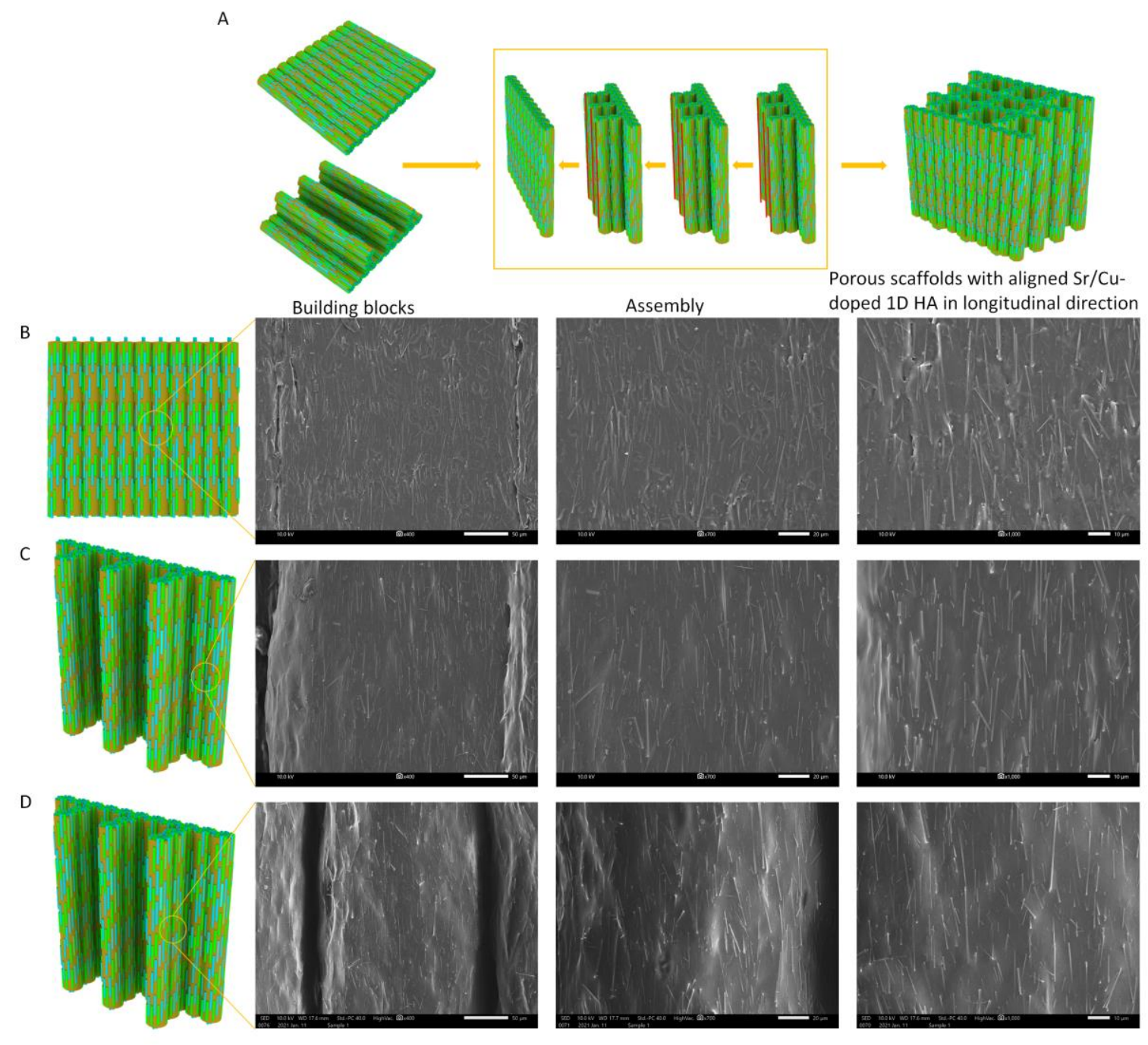

Figure S13. (A) Fabrication process of the assembled Sr/Cu-doped 1D HA/PDLA porous scaffolds with all $\mathrm{Sr} / \mathrm{Cu}$-doped 1D HA aligned in the longitudinal direction. (B to D) SEM micrographs of the aligned $\mathrm{Sr} / \mathrm{Cu}$-doped 1D HA in the building blocks. 

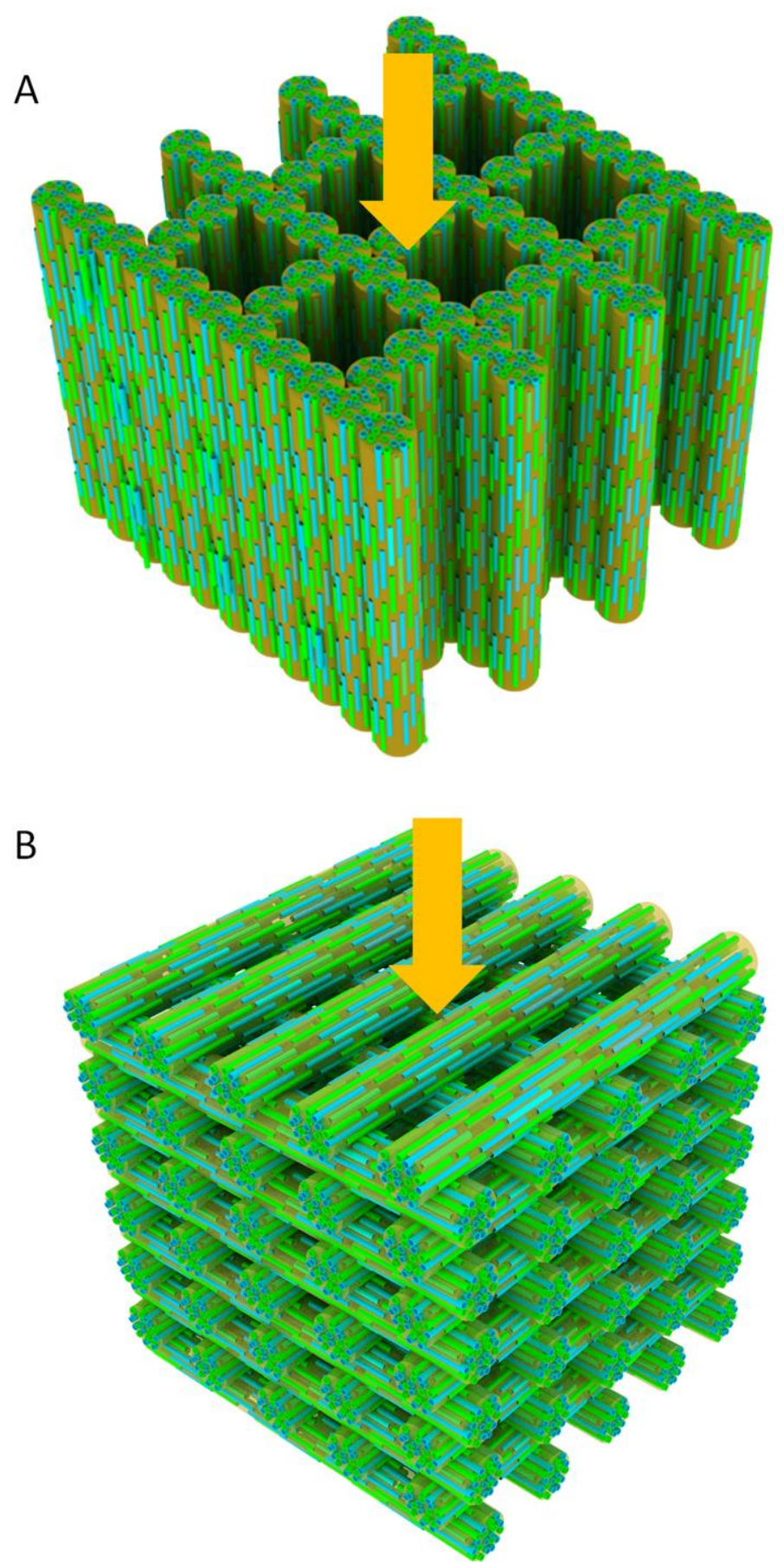

Figure S14. Schematic illustration of the loading process during compressive test of assembled Sr/Cu-doped 1D HA/PDLA porous scaffolds (A) and fully printed Sr/Cu-doped 1D HA/PDLA porous scaffolds (B). 

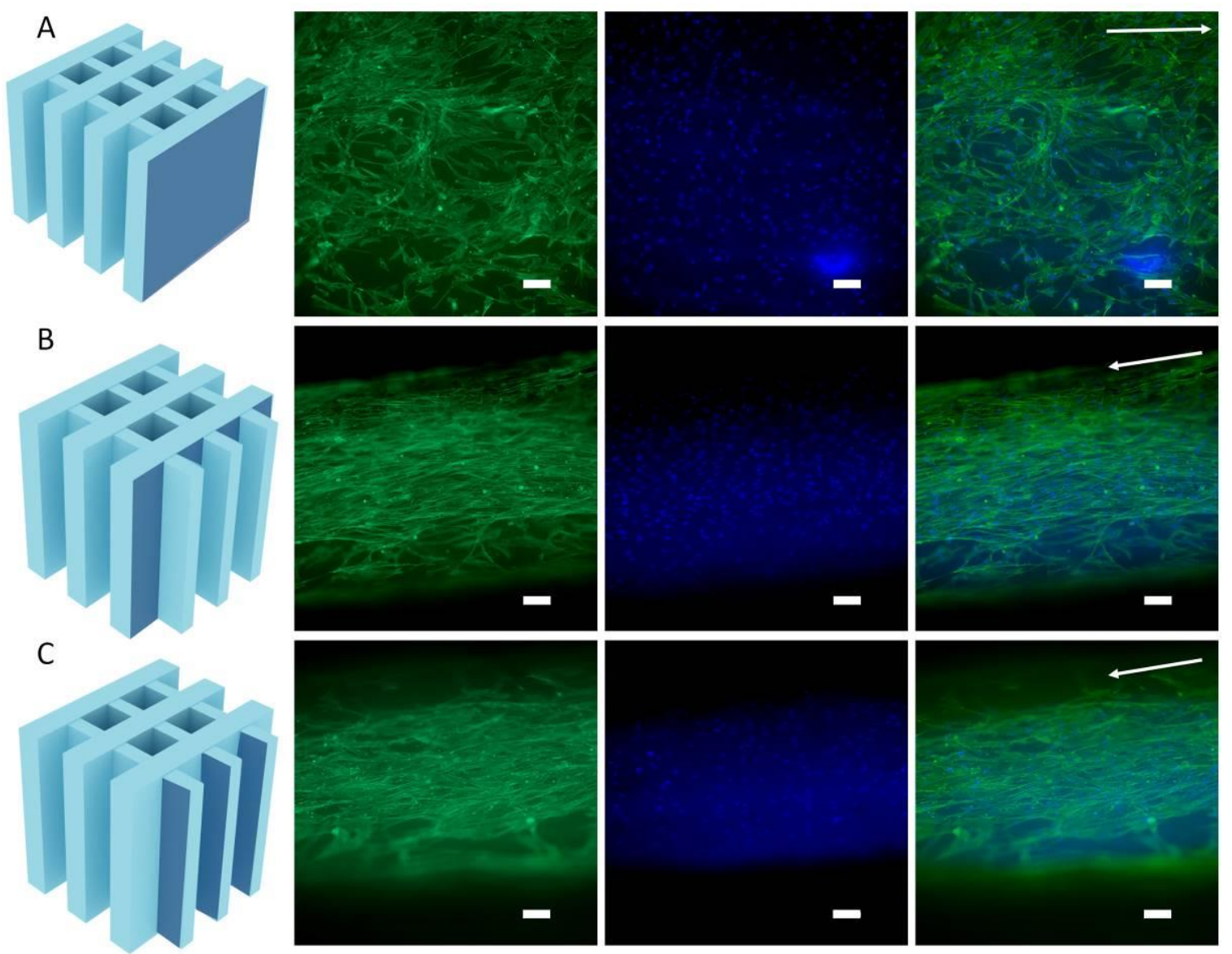

Figure S15. (A to C) Fluorescence microscopy images of actin cytoskeleton (phalloidin: green) and nuclei (DAPI: blue) of hMSCs cultured on the assembled Nano HA/PDLA porous scaffolds for 4 days. Arrows indicate the longitudinal direction. Scale bar: $100 \mu \mathrm{m}$. 

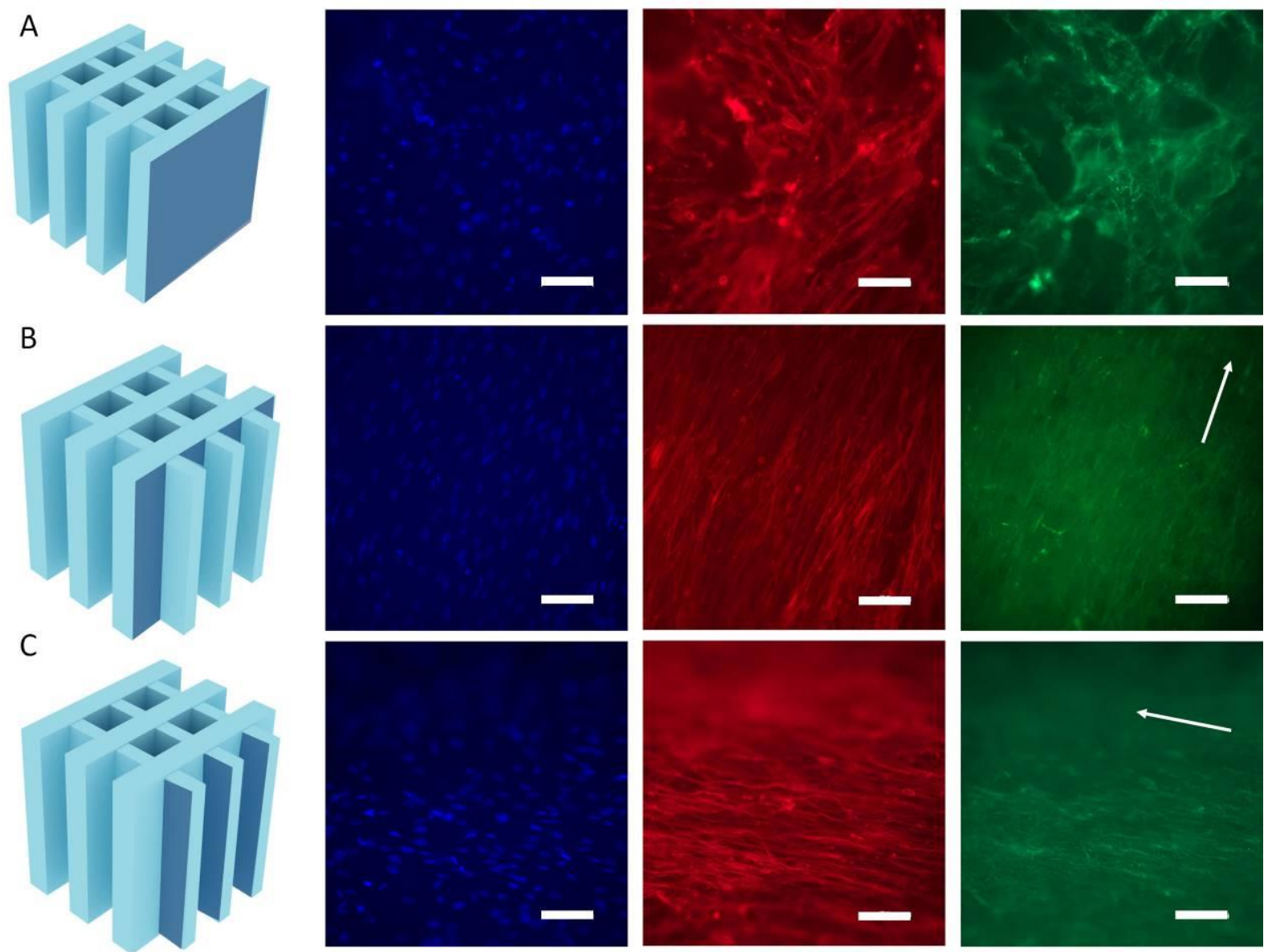

Figure S16. (A to C) Fluorescence microscopy images of nuclei (DAPI: blue), actin cytoskeleton (phalloidin: red) and type I collagen (green) of hMSCs cultured on the assembled Nano HA/PDLA porous scaffolds for 14 days. Arrows indicate the direction of the alignment. Scale bar: $100 \mu \mathrm{m}$. 
Table S1. UTS and Young's modulus of biomimetic Sr/Cu-doped 1D HA/PCL composite with $30 \mathrm{wt} \%$ and $50 \mathrm{wt} \% \mathrm{Sr} / \mathrm{Cu}$-doped 1D HA, and Nano HA/PCL composite with $30 \mathrm{wt} \%$ and 50 wt \% Nano HA, relative to those of pure PCL.

\begin{tabular}{|c|c|c|}
\hline & UTS & Young's modulus \\
\hline PCL-30- Sr/Cu-doped 1D HA & $+86 \%$ & $+55.7 \%$ \\
\hline PCL-30-Nano HA & $+5.5 \%$ & $+11.4 \%$ \\
\hline PCL-50- Sr/Cu-doped 1D HA & $+50.34 \%$ & $+30.8 \%$ \\
\hline PCL-50-Nano HA & $-23.4 \%$ & $+12.1 \%$ \\
\hline
\end{tabular}

Table S2. Compressive strength (at 5\% strain) and compressive modulus of biomimetic $\mathrm{Sr} / \mathrm{Cu}-$ doped 1D HA/PCL composite with 30 wt \% and 50 wt \% Sr/Cu-doped 1D HA, and Nano HA/PCL composite with 30 wt $\%$ and 50 wt \% Nano HA, relative to those of pure PCL.

\begin{tabular}{|c|c|c|}
\hline & Compressive strength & Compressive modulus \\
\hline PCL-30- Sr/Cu-doped 1D HA & $+267.3 \%$ & $+408.3 \%$ \\
\hline PCL-30-Nano HA & $+119 \%$ & $+139.7 \%$ \\
\hline PCL-50- Sr/Cu-doped 1D HA & $+258.3 \%$ & $+394.7 \%$ \\
\hline PCL-50-Nano HA & $+180 \%$ & $+182.2 \%$ \\
\hline
\end{tabular}

Table S3. UTS and Young's modulus of biomimetic Sr/Cu-doped 1D HA/PDLA composite with 25 wt \% Sr/Cu-doped 1D HA and Nano HA/PDLA composite with 25 wt \% Nano HA, relative to those of pure PDLA. 


\begin{tabular}{|c|c|c|}
\hline & UTS & Young's modulus \\
\hline PDLA-25- Sr/Cu-doped 1D HA & $+102 \%$ & $+120.6 \%$ \\
\hline PDLA-25-Nano HA & $-0.08 \%$ & $+29.6 \%$ \\
\hline
\end{tabular}

Table S4. Compressive strength and compressive modulus of biomimetic $\mathrm{Sr} / \mathrm{Cu}$-doped 1D HA/PDLA composite with 25 wt \% Sr/Cu-doped 1D HA and Nano HA/PDLA composite with 25 wt $\%$ Nano HA, relative to those of pure PDLA.

\begin{tabular}{|c|c|c|}
\hline & Compressive strength & Compressive modulus \\
\hline PDLA-25- Sr/Cu-doped 1D HA & $+63.1 \%$ & $+85 \%$ \\
\hline PDLA-25-Nano HA & $-24.8 \%$ & $-7.4 \%$ \\
\hline
\end{tabular}

Table S5. Comparison of reinforcement efficiency of tensile strength of HA/PLA composites between this work and other literature.

\begin{tabular}{|c|c|c|}
\hline HA content $($ vol \%) & $\begin{array}{r}\text { Change of tensile strength relative } \\
\text { to polymer alone }\end{array}$ & \\
\hline $5 \%$ & $-13.8 \%$ & 4 \\
$10 \%$ & $-38 \%$ & \\
$15 \%$ & $-44.8 \%$ & \\
$20 \%$ & $-53.4 \%$ & \\
$25 \%$ & $-55.2 \%$ & \\
\hline $4.2 \%$ & $+13.5 \%$ & \\
$8.9 \%$ & $+27.0 \%$ & \\
\hline $14.5 \%$ & $+2.7 \%$ & \\
\hline
\end{tabular}




\begin{tabular}{|c|c|c|}
\hline $2.0 \%$ & $-13.4 \%$ & \multirow{4}{*}{6} \\
\hline $4.1 \%$ & $-15.9 \%$ & \\
\hline $6.5 \%$ & $-22.6 \%$ & \\
\hline $8.9 \%$ & $-27.4 \%$ & \\
\hline $4.2 \%$ & $+5.3 \%$ & 7 \\
\hline $4.2 \%$ & $-9.9 \%$ & \multirow{3}{*}{8} \\
\hline $8.9 \%$ & $-19.8 \%$ & \\
\hline $14.4 \%$ & $-28.6 \%$ & \\
\hline $0.4 \%$ & $-9.4 \%$ & \multirow{2}{*}{9} \\
\hline $1.0 \%$ & $-11.3 \%$ & \\
\hline $1.2 \%$ & $+5.1 \%$ & \multirow{4}{*}{10} \\
\hline $2.0 \%$ & $+6.6 \%$ & \\
\hline $4.2 \%$ & $+8.3 \%$ & \\
\hline $6.5 \%$ & $+3.0 \%$ & \\
\hline $3.9 \%$ & $-5.3 \%$ & \multirow{5}{*}{11} \\
\hline $8.3 \%$ & $-28.3 \%$ & \\
\hline $13.5 \%$ & $-52.6 \%$ & \\
\hline $19.5 \%$ & $-63.5 \%$ & \\
\hline $26.7 \%$ & $-82.4 \%$ & \\
\hline $1.7 \%$ & $+5.6 \%$ & \multirow{5}{*}{12} \\
\hline $2.5 \%$ & $-4.2 \%$ & \\
\hline $4.3 \%$ & $-12.7 \%$ & \\
\hline $6.7 \%$ & $-14.1 \%$ & \\
\hline $9.3 \%$ & $-15.5 \%$ & \\
\hline $11.6 \%$ & $+102.2 \%$ & This work \\
\hline
\end{tabular}


Table S6. Comparison of compressive strength of HA/PLA composites between this work and other literature.

\begin{tabular}{|c|c|c|c|}
\hline $\begin{array}{l}\text { Young's modulus } \\
(\mathrm{GPa})\end{array}$ & Compressive strength (MPa) & $\begin{array}{c}\text { Molecular weight of } \\
\text { PLA (Mw) }\end{array}$ & Ref \\
\hline $\begin{array}{l}2.3 \\
3.6\end{array}$ & $\begin{array}{l}109 \\
155\end{array}$ & $200 \mathrm{~K}$ & 13 \\
\hline 2.4 & 115 & $202 \mathrm{~K}$ & 14 \\
\hline $\begin{array}{l}3.8 \\
5.6\end{array}$ & $\begin{array}{l}115 \\
125\end{array}$ & $104 K$ & 15 \\
\hline $\begin{array}{l}1.08 \\
1.31 \\
1.21\end{array}$ & $\begin{array}{c}109.1 \\
110.3 \\
76.4\end{array}$ & $680 \mathrm{~K}$ & 11 \\
\hline $\begin{array}{l}0.789 \\
0.82 \\
0.834 \\
0.811\end{array}$ & $\begin{array}{l}88.9 \\
93.7 \\
93.3 \\
89.2\end{array}$ & $223 \mathrm{~K}$ & 10 \\
\hline $\begin{array}{l}1.3 \\
1.6 \\
1.7 \\
1.8\end{array}$ & $\begin{array}{l}78 \\
73 \\
84 \\
88\end{array}$ & $60 \mathrm{~K}-80 \mathrm{~K}$ & 16 \\
\hline 6.1 & 116 & $\sim 60 \mathrm{k}$ & This work \\
\hline
\end{tabular}




\section{REFERENCES}

(1) Wu, C. T.; Zhou, Y. H.; Lin, C. C.; Chang, J.; Xiao, Y. Strontium-Containing Mesoporous Bioactive Glass Scaffolds with Improved Osteogenic/Cementogenic Differentiation of Periodontal Ligament Cells for Periodontal Tissue Engineering. Acta Biomater. 2012, 8 (10), 3805-3815.

(2) Wu, C. T.; Zhou, Y. H.; Xu, M. C.; Han, P. P.; Chen, L.; Chang, J.; Xiao, Y. CopperContaining Mesoporous Bioactive Glass Scaffolds with Multifunctional Properties of Angiogenesis Capacity, Osteostimulation and Antibacterial Activity. Biomaterials 2013, 34 (2), 422-433.

(3) Rustom, L. E.; Poellmann, M. J.; Johnson, A. J. W. Mineralization in Micropores of Calcium Phosphate Scaffolds. Acta Biomater. 2019, 83, 435-455.

(4) Bleach, N. C.; Nazhat, S. N.; Tanner, K. E.; Kellomäki, M.; Törmälä, P. Effect of Filler Content on Mechanical and Dynamic Mechanical Properties of Particulate Biphasic Calcium Phosphate-Polylactide Composites. Biomaterials 2002, 23 (7), 1579-1585.

(5) Wan, Y.; Wu, C.; Xiong, G.; Zuo, G.; Jin, J.; Ren, K.; Zhu, Y.; Wang, Z.; Luo, H. Mechanical Properties and Cytotoxicity of Nanoplate-Like Hydroxyapatite/Polylactide Nanocomposites Prepared by Intercalation Technique. J. Mech. Behav. Biomed. 2015, 47, 2937.

(6) Akindoyo, J. O.; Beg, M. D. H.; Ghazali, S.; Heim, H. P.; Feldmann, M. Effects of Surface Modification on Dispersion, Mechanical, Thermal and Dynamic Mechanical Properties of Injection Molded PLA-Hydroxyapatite Composites. Compos. Part A Appl. Sci. Manuf. 2017, 103, 96-105.

(7) Akindoyo, J. O.; Beg, M. D. H.; Ghazali, S.; Heim, H. P.; Feldmann, M. Impact Modified PLA-Hydroxyapatite Composites - Thermo-Mechanical Properties. Compos. Part A Appl. Sci. Manuf. 2018, 107, 326-333.

(8) Ferri, J.; Jordá, J.; Montanes, N.; Fenollar, O.; Balart, R. Manufacturing and Characterization of Poly(Lactic Acid) Composites with Hydroxyapatite. J. Thermoplast. Compos. 2018, 31 (7), 865-881.

(9) Pérez, C. J.; Eisenberg, P.; Bernal, C.; Pérez, E. Mechanical Evaluation of Polylactic Acid (PLA) Based Composites Reinforced with Different Calcium Phosphates. Mater. Res. Express 2018, 5 (10), 105304.

(10) Huang, Z. H.; Wan, Y. Z.; Peng, M. X.; Yang, Z. W.; Luo, H. L. Incorporating NanoplateLike Hydroxyapatite into Polylactide for Biomimetic Nanocomposites via Direct Melt Intercalation. Compos. Sci. and Technol. 2020, 185.

(11) Aydin, E.; Planell, J. A.; Hasirci, V. Hydroxyapatite Nanorod-Reinforced Biodegradable Poly(L-Lactic Acid) Composites for Bone Plate Applications. J. Mater. Sci. Mater. Med. 2011, 22 (11), 2413-2427.

(12) Hong, Z.; Zhang, P.; He, C.; Qiu, X.; Liu, A.; Chen, L.; Chen, X.; Jing, X. Nano-Composite of Poly(L-Lactide) and Surface Grafted Hydroxyapatite: Mechanical Properties and Biocompatibility. Biomaterials 2005, 26 (32), 6296-6304.

(13) Zhang, C. Y.; Lu, H.; Zhuang, Z.; Wang, X. P.; Fang, Q. F. Nano-Hydroxyapatite/Poly(LLactic Acid) Composite Synthesized by a Modified in Situ Precipitation: Preparation and Properties. J. Mater. Sci. Mater. Med. 2010, 21 (12), 3077-3083.

(14) Shikinami, Y.; Okuno, M. Bioresorbable Devices Made of Forged Composites of Hydroxyapatite (HA) Particles and Poly-L-Lactide (PLLA): Part I. Basic Characteristics. Biomaterials 1999, 20 (9), 859-877.

(15) Gay, S.; Arostegui, S.; Lemaitre, J. Preparation and Characterization of Dense Nanohydroxyapatite/PLLA Composites. Mater. Sci. Eng. C 2009, 29 (1), 172-177. 
(16) Wu, D.; Spanou, A.; Diez-Escudero, A.; Persson, C. 3D-Printed PLA/HA Composite Structures as Synthetic Trabecular Bone: A Feasibility Study Using Fused Deposition Modeling. J. Mech. Behav. Biomed. 2020, 103. 